\title{
Calculations of Magnetic Exchange Interactions in Mott-Hubbard Systems
}

\author{
Xiangang Wan ${ }^{1,2}$, Quan Yin ${ }^{1}$, Sergej Y. Savrasov ${ }^{1}$ \\ ${ }^{1}$ Department of Physics, University of California, Davis, One Shields Ave, Davis, CA 95616 and \\ ${ }^{2}$ National Laboratory of Solid State Microstructures and Department of Physics, Nanjing University, Nanjing 210093, China
}

(Dated: March 22, 2022)

\begin{abstract}
An efficient method to compute magnetic exchange interactions in systems with strong correlations is introduced. It is based on a magnetic force theorem which evaluates linear response due to rotations of magnetic moments and uses a novel spectral density functional framework combining our exact diagonalization based dynamical mean field and local density functional theories. Applications to spin waves and magnetic transition temperatures of $3 d$ metal mono-oxides as well as high- $\mathrm{T}_{c}$ superconductors are in good agreement with experiment.
\end{abstract}

PACS numbers: 71.27.+a, 75.30.Et, 71.15.-m, 75.10.-b

Obtaining a quantitative theory of magnetic materials spanning from itinerant to atomic limit, above and below their temperatures of magnetic ordering has been a theoretical challenge for many years [1]. By now itinerant magnets are well described by local spin density approximation (LSDA) of density functional theory (DFT) 2], where methodologies based on spin-spiral frozenmagnon technique [3], the use of magnetic force theorem 4] via an evaluation of linear response due to rotations of magnetic moments as well as spin dynamics calculations [5] have allowed to access a great variety of physical properties such as spin wave spectra, magnetic ordering temperatures, interatomic exchange constants, dynamical susceptibilities, etc $[\mathbf{6}, 7,6,8,9,10]$.

However, there is large class of systems where calculations of exchange interactions is still a challenging theoretical problem. These are strongly correlated systems like high- $\mathrm{T}_{c}$ superconductors or atomic magnets where the on-site Coulomb interaction $U$ is comparable or larger than the bandwidth. In cases where magnetic ordering and/or lattice distortions result in a non-degenerate equilibrium state, techniques such as $\mathrm{LDA}+\mathrm{U}$ 11 or GW 12 have been applied to describe spectroscopy, magnetic moments, and even spin wave spectra of systems such as $\mathrm{MnO}$ [13]. However, in general, excitation spectra of strongly correlated systems are not representable by single Slater determinants and show such features as atomic multiplets [14], Zhang-Rice singlets 15., Kondo resonances, etc. In Mott-Hubbard insulators the energy gap is much larger than the magnetic ordering temperature above which a local moment regime takes place, i.e. the system becomes paramagnetic but remains insulating. These properties cannot be accessed either by static mean field approaches such as LSDA or $\mathrm{LDA}+\mathrm{U}$ or by perturbation theory over the Coulomb interaction such as GW. While versions of static 16] as well as dynamic [17] coherent potential approximations have been introduced in the past to access disordered local moment regime, developing a generalized framework having a capability to compute exchange interactions in both itinerant and atomic limits as well as in many in- termediated cases would open new opportunities in computational design of new magnetic materials.

In the present work we develop a novel approach which is capable to deal with this problem. Our method is based on LDA+Dynamical Mean Field Theory (DMFT) [18], a recently developed electronic structure method describing correctly both itinerant and atomic limits and accessing ordered and disordered moment regimes on equal footing. This is achieved by treating correlated electrons with frequency dependent self-energies deduced from solving corresponding Anderson impurity problem (AIM) subjected to a self-consistency condition. Conveniently formulated using a spectral density functional 18], this LDA+DMFT method incorporates realistic band structure effects and has already helped to solve several long-standing problems, e.g. temperaturedependent magnetism of $\mathrm{Fe}$ and $\mathrm{Ni}[19]$, volume collapse in $\mathrm{Ce} 20$, and $\mathrm{Pu} 21$, as well as electronic structure of doped Mott insulators 22.

In order to deduce exchange constants for general wave vector $\mathbf{q}$ we utilize a linear response based magnetic force theorem [4, 23] and demonstrate the accuracy of our method on several Mott-Hubbard insulators including late transition metal oxides as well as parent materials of high- $\mathrm{T}_{c}$ superconductors. To solve the impurity problem which is the nucleus of the dynamical mean field algorithm we apply a newly implemented cluster exact diagonalization method and calculate self-consistently local Green functions, self-energies and static linear response functions. Both the deduced spin wave spectra as well as magnetic ordering temperatures evaluated using a Monte Carlo simulations of the mapped Heisenberg Hamiltonians are found in good agreement with experiment.

Our implementation is based on a most recent manybody band structure algorithm [24] which allows us to avoid computationally expensive solution of the Dyson equation $\left[\omega-H_{0}(\mathbf{k})-\Sigma(\omega)\right] G(\mathbf{k}, \omega)=1$ for the electronic Green function $G(\mathbf{k}, \omega)$ at a large grid of frequencies $\omega$. This is achieved by assuming a pole expansion for the 
self-energy

$$
\Sigma(\omega)=\Sigma(\infty)+\sum_{i} V_{i}^{+}\left(\omega-P_{i}\right)^{-1} V_{i}
$$

so that the entire problem is reduced to a matrix equation with an energy-independent Hamiltonian

$$
\left(\begin{array}{cc}
\omega-H_{0}(\mathbf{k})-\Sigma(\infty) & V^{+} \\
V & P
\end{array}\right) \mathcal{G}(\mathbf{k}, \omega)=I
$$

where $I$ is the unit matrix and an auxiliary Green function $\mathcal{G}_{\alpha \beta}(\mathbf{k}, \omega)$ is a matrix in the space of poles, while the physical Green function $G(\mathbf{k}, \omega)$ corresponds to the first element of $\mathcal{G}(\mathbf{k}, \omega)$. In Eq. (11) weights $V_{i}^{+}, V_{i}$ and poles $P_{i}$ can be viewed as matrices which provide a best fit to real $\Sigma(\omega)$.
It is remarkable that the present procedure allows us to use an expression for the interatomic exchange constants similar to a linear response formula derived within DFT [4]. Consider second-order change in the total energy related to the rotations of the magnetic moments appeared at sites $R+\tau$ and $R^{\prime}+\tau^{\prime}$ of the lattice (here $R$ are the lattice translations and $\tau$ are the atoms in the basis). The local magnetic field $\mathbf{B}_{\tau}$ at every atomic site $\tau$ is approximately described by the values of the self-energy taken at $\omega=\infty$ [for example, $B_{\tau}^{z}=\Sigma_{\tau}^{\uparrow \uparrow}(\infty)-\Sigma_{\tau}^{\downarrow \downarrow}(\infty)$ ]. Thus, according to the magnetic force theorem which assumes a rigid rotation of atomic spin, a linear response theory expresses the interatomic exchange constants in the form

$$
J_{\tau R \tau^{\prime} R^{\prime}}^{\alpha \beta}=\sum_{\mathbf{q}} \sum_{\mathbf{k} j j^{\prime}} \frac{f_{\mathbf{k} j}-f_{\mathbf{k}+\mathbf{q} j^{\prime}}}{\epsilon_{\mathbf{k} j}-\epsilon_{\mathbf{k}+\mathbf{q} j^{\prime}}}\left\langle\psi_{\mathbf{k} j}\left|\left[\sigma \times \mathbf{B}_{\tau}\right]_{\alpha}\right| \psi_{\mathbf{k}+\mathbf{q} j^{\prime}}\right\rangle\left\langle\psi_{\mathbf{k}+\mathbf{q} j^{\prime}}\left|\left[\sigma \times \mathbf{B}_{\tau^{\prime}}\right]_{\beta}\right| \psi_{\mathbf{k} j}\right\rangle e^{i \mathbf{q}\left(\mathbf{R}-\mathbf{R}^{\prime}\right)}
$$

Here, $\sigma$ is the Pauli matrix while the one-electron energy bands $\epsilon_{\mathbf{k} j}$ and quasiparticle wave functions $\psi_{\mathbf{k} j}$ appear as the solutions of the equation (2), when using quasiparticle representation for the Green function $\mathcal{G}(\mathbf{k}, \omega)$ in the form.

$$
\mathcal{G}(\mathbf{k}, \omega)=\sum_{j} \frac{\psi_{\mathbf{k} j}^{+} \psi_{\mathbf{k} j}}{\omega-\epsilon_{\mathbf{k} j}}
$$

While viewed non-interacting like, this formula indeed contains major information about many-body features in the excitation spectrum. In particular, multiplet transitions as well as delocalized parts of the electronic states are represented by separate "energy bands" $\epsilon_{\mathbf{k} j}$ including its $\mathbf{k}$ dispersion which is borrowed from the noninteracting Hamiltonian $H_{0}(\mathbf{k})$. Thus, genuine redistribution of spectral weight driven by the many-body interactions is correctly captured by the present method which will give an important feedback on the calculated exchange interactions.

There are two essential approximations which are made to make the theory computationally tractable. As has been discussed recently [23], the magnetic force theorem can be introduced for a Lattinger-Ward functional which would involve calculations of full frequency dependent integrals between the self-energies and the Green functions. The present method utilizes (i) the Hartree Fock values for the local magnetic fields, and (ii) rational fit, i.e. Eq. (11), to the self-energy, which allows us to perform all frequency sums analytically while retaining all major many-body multiplet features of the spectrum in the convenient linear response expression (3).

To illustrate the method we consider several transition-metal oxides $\mathrm{MnO}, \mathrm{FeO}, \mathrm{CoO}, \mathrm{NiO}$ as well as parent high- $\mathrm{T}_{c}$ compound $\mathrm{CaCuO}_{2}$. All these materials are antiferromagnetic insulators with an energy gap of a few $\mathrm{eV}$ and Neel temperatures $\mathrm{T}_{N}$ of a few hundred K. Staying below $\mathrm{T}_{N}$ it is well known that LSDA significantly underestimates the band gap of $\mathrm{MnO}$ and $\mathrm{NiO}$ and fails to predict insulating character for $\mathrm{FeO}, \mathrm{CoO}$ and $\mathrm{CaCuO}_{2}$. The $\mathrm{LDA}+\mathrm{U}$ corrects for these failures but needs to assume a symmetry breaking for $\mathrm{FeO}$ and CoO. It is clear that being a Hartree Fock approximation the LDA $+\mathrm{U}$ would converge to a single Slater determinant ground state, while in many cases either degeneracy of the latter or proximity of low-lying excited states needs to be included in statistical averagings for the oneelectron Green functions. All static mean field theories would necessarily fail to describe paramagnetic insulating behavior. On the other hand, the LDA+DMFT is a method valid both in ordered and local moment regimes. Here, we consider the $d$ electrons of transition metal elements as strongly correlated thus requiring dynamical treatment using DMFT. The $s$ and $p$ electrons are assumed to be weakly correlated and well described by the LDA Hamiltonian $H_{L D A}(\mathbf{k})$ including the full potential terms of the linear muffin-tin orbital (LMTO) method 25]. To consider relativistic effects, the spin-orbit coupling is taken into account in all cases. To obtain the one-particle potential $H_{0}(\mathbf{k})=H_{L D A}(\mathbf{k})-V_{d c}$ entering (2) we subtract the double counting term $V_{d c}$ as pre- 
scribed by Ref. [11]. We use the experimental lattice structure for all materials.

In order to solve the Anderson impurity problem we implement cluster exact diagonalization (ED) method. For transition metal mono-oxides the clusters are chosen to include $d$ orbitals of transition metal ions hybridized with oxygen $p$ orbitals in the octahedral environment. It has been known for many years that such cluster exact diagonalizations provide a good description of photoemission spectra in these materials 26, 27]. For the 2D system, such as $\mathrm{CaCuO}_{2}$ this is reduced to a $\mathrm{Cu} d$ orbital surrounded by an oxygen square. This treatment allows us to capture both an effect of atomic multiplets and of the Zhang-Rice singlet [15] being the lowest lying excitation of undoped high- $\mathrm{T}_{c}$ 's. During iterations towards self-consistency, the Anderson impurity problem is exact diagonalized each time with the positions of $3 d$ levels, the $d-p$ hybridization matrix elements as well as the $\mathrm{O} 2 p$ levels are extracted from the $H_{L D A}(\mathbf{k})$. The latter being a density functional is allowed to recompute and readjust the parameters of AIM. The values of the Coulomb interaction $U$ as well as the Hund's exchange $J$ were obtained earlier by the constrained LDA calculation [28] and kept fixed throughout the calculation. The AIM gives access to the frequency dependent self-energies for the $d$ electrons which are then rationally approximated assuming three poles fit in the formula (1).

Our calculated ground state properties including magnetic moments and energy gaps are found to be in good agreement with experiment both below and above magnetic ordering temperatures. Below $T_{N}$ this result is in accord with previous LDA+U studies [28] and our numerical results indeed show that dynamical correlations only marginally influence values of magnetic moments in cases such as $\mathrm{MnO}, \mathrm{NiO}$ and $\mathrm{CaCuO}_{2}$. On the other hand, for $\mathrm{CoO}$ and $\mathrm{FeO}$, their $\mathrm{t}_{2 g}$ bands are only partially occupied and the ground states become degenerate. Therefore small value of spin-orbital coupling has a large effect on an appeared orbital moments which are evaluated to be 0.36 and $1.02 \mu_{B}$ in our cluster exact diagonalized LDA+DMFT calculations for $\mathrm{FeO}$ and $\mathrm{CoO}$ respectively. Note that the $\mathrm{LDA}+\mathrm{U}$ would be capable recover the insulating character only by assuming symmetry lowered orbitally ordered solution.

We now discuss our predictions for the calculated Neel temperatures. Based on the self-consistently obtained local Green functions and self-energies we evaluate the interatomic exchange constants as the integral over the q space using $(6,6,6)$ reciprocal lattice grid and utilize Monte-Carlo simulations 7] of the correspondingly mapped Heisenberg Hamiltonians to find $\mathrm{T}_{N}$. Our results are given in Table I, where together with our most accurate exact diagonalization based simulations we also list the predictions using LSDA and less accurate impurity solvers: single atom exact diagonalization known as Hubbard I solver [14], and the Hartree-Fock approx- imation to atomic self-energy known as $\mathrm{LDA}+\mathrm{U}$. It is well known that LSDA underestimates the energy gap, and as a result it significantly overestimates the magnetic transition temperatures as seen in Table I. (Since for $\mathrm{FeO}, \mathrm{CoO}$ and $\mathrm{CaCuO}_{2}$, LSDA converges to a completely wrong metallic state, we omit quoting those predictions). The LDA $+U$ method considers the effect of $U$ in a static way, and this partially cures the shortcomings of LSDA making $T_{N}$ smaller but still much larger than the experimental ones. This conclusion is in agreement with the previously calculated results for $\mathrm{MnO}$ [13]. Hubbard I approximation uses exact diagonalization for atomic $d$-shell and deduces frequency dependent self-energy, which further reduces $\mathrm{T}_{N}$ for all transition-metal oxides. The best results are seen to be obtained by allowing $d$-electrons to fluctuate between the bath and impurity as prescribed by the cluster exact diagonalization calculation. This effect is missing in the Hubbard I solver and seriously affects the electronic structure of studied materials as, for example, for $\mathrm{CaCuO}_{2}$ it redistributes the spectral weight by bringing such features as the well known Zhang-Rice singlet [15]. In an extreme situation, where the hybridization is much larger than the local Coulomb $U$, the magnetization would eventually disappear due to strong fluctuation in the number of $d$-electrons at the impurity site. So it is easy to understand why the cluster ED method gives smaller magnetic transition temperatures which are now closer to the experiment.

It is interesting to discuss the physical reasons why $\mathrm{T}_{N}$ decreases when going from $\mathrm{NiO}$ to $\mathrm{MnO}$. These MottHubbard insulators show almost atomic values of magnetic moments $M=10-n$ corresponding to $d^{n}$ configurations, which would under assumption of the same antiferromagnetic exchange constant $J_{A F}$ mean that the ordering temperatures should increase with increasing the moments. However, $J_{A F}$ will decrease significantly due to the change in the lattice parameter. Also, during evaluation of $\mathrm{T}_{N}$ we need to account for the quantum averaging for atomic spins directions which gives a prefactor $S(S+1) / S^{2}$ deviating from 1 for small $S$. To sort out these effects we first performed a sample calculation for $\mathrm{NiO}$ with the expanded lattice constant of $\mathrm{MnO}$. The $\mathrm{T}_{N}$ for $\mathrm{NiO}$ has dropped from $519 \mathrm{~K}$ to $327 \mathrm{~K}$ in this case. Second, since $S_{N i}=1$ while $S_{M n}=5 / 2$, the prefactor $S(S+1) / S^{2}$ would account for a $40 \%$ difference so that $327 \times 1.4 / 2=229 \mathrm{~K}$ is the Neel temperature that we need to compare with our predicted $\mathrm{T}_{N}=172 \mathrm{~K}$ for $\mathrm{MnO}$. The residual discrepancy can be attributed to different exchange splittings which also affects $J_{A F}$ as pointed out earlier [31].

We now discuss our calculated spin-wave dispersions along major symmetry directions in the Brillouin Zone. We illustrate this calculation on a case of $\mathrm{NiO}$ for which the magnon spectra have been measured long time ago 32. Fig. 1 shows the results of our simulations using several levels of approximations. In accord with our pre- 
TABLE I: Comparison between calculated using various approximations and experimental magnetic transition temperatures (in K) in selected Mott-Hubbard systems. Hubbard I and Cluster ED denote the results of LDA+DMFT calculations using Hubbard I and cluster exact diagonalization impurity solver.

\begin{tabular}{|c|c|c|c|c|c|}
\hline & LSDA & $\mathrm{LDA}+\mathrm{U}$ & Hubbard I & Cluster ED & Exp. \\
\hline $\mathrm{MnO}$ & 423 & 240 & 180 & 172 & $122^{a}$ \\
\hline $\mathrm{FeO}$ & - & 344 & 297 & 211 & $198^{a}$ \\
\hline $\mathrm{CoO}$ & - & 407 & 356 & 300 & $291^{a}$ \\
\hline $\mathrm{NiO}$ & 965 & 603 & 542 & 519 & $523^{a}$ \\
\hline $\mathrm{CaCuO}_{2}$ & - & 765 & 698 & 602 & $537^{b}$ \\
\hline
\end{tabular}

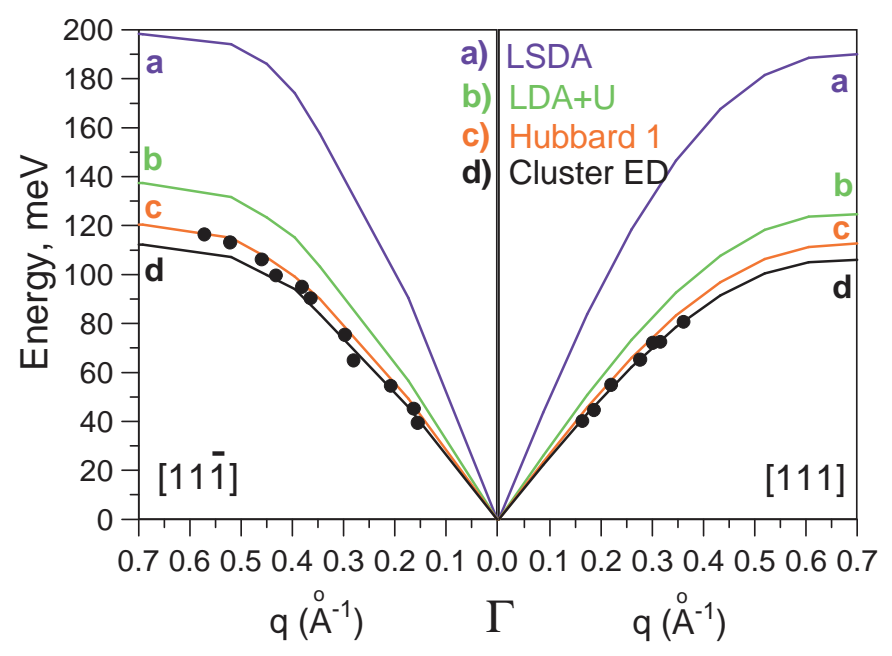

FIG. 1: Theoretical spin-wave dispersions for $\mathrm{NiO}$ calculated by LSDA, LDA+U, Hubbard I and Cluster Exact Diagonalization (ED) diagonalization impurity solvers in comparison with the experiment [32].

dictions for the Neel temperatures, the spin waves are seriously overestimated by the LSDA theory but get closer to the experiment once correlations are taken into account. The best accuracy is achieved when using the cluster exact diagonalization as the impurity solver in the LDA+DMFT calculation which demonstrates the importance of many body redistribution of the spectral weight on the calculated exchange integrals.

In summary, based on our newly implemented cluster exact diagonalization LDA+DMFT calculation, we presented a linear response method to calculate the exchange interaction parameters of strongly correlated systems valid as long as mapping of total energy functional to rigid spin based Heisenberg Hamiltonians makes sense. By using the rational interpolation for the self-energy, our approach is very efficient, and this has allowed us to describe quantitatively spin-wave dispersions and magnetic transition temperatures of several realistic MottHubbard insulators with many atoms per unit cell. Applications to metallic systems are more challenging as they may need much larger clusters to account for such subtle effects as, e.g., the Kondo screening, and will be carried out in the future work.

We acknowledge useful conversations with G. Kotliar and A. Lichtenstein. The work was supported by NSF DMR grants 0608283 and 0606498.

[1] For a review, see, e.g., J. Kübler, Theory of Itinerant Electron, Magnetism (Oxford University Press, 2000).

[2] For a review, see, e.g., Theory of the Inhomogeneous Electron Gas, ed. by S. Lundqvist, S. H. March (Plenum, New York, 1983).

[3] For a review, see, e.g., L. M. Sandratskii, Adv. Phys. 47, 91 (1998).

[4] A. I. Liechtenstein, M. I. Katsnelson, V. P. Antropov, V. A. Gubanov, J. Mag. Mag. Mater. 67, 65 (1987), P. Bruno, Phys. Rev. Lett. 90, 087205 (2003).

[5] V. P. Antropov, M. I. Katsnelson, M. van Schilfgaarde, B. N. Harmon, Phys. Rev.Lett. 75, 729 (1995); V.P. Antropov, J. Mag. Mag. Mater. 262, L192 (2003).

[6] S. V. Halilov, A. Y. Perlov, P. M. Oppeneer, H. Eschrig, Europhys. Lett. 39, 91 (1997); O. Grotheer, C. Ederer, and M. Fähnle, Phys. Rev. B 63, 100401(R) (2001).

[7] N.M. Rosengaard and B. Johansson, Phys. Rev. B 55, 14975 (1997).

[8] M. Uhl and J. Kübler, Phys. Rev. Lett. 77, 334 (1996); M. van Schilfgaarde and F. Herman, Phys. Rev. Lett. 71, 1923 (1993); M. Pajda, J. Kudrnovsky, I Turek, V. Drchal, P. Bruno, Phys. Rev. B 64, 174402 (2001).

[9] J. Callaway, A. K. Chatterjee, S. P. Singhal, A. Ziegler, Phys. Rev. B 28, 3818 (1983); E. Stenzel, H. Winter, J. Phys. F: Met. Phys. 16, 1789 (1986); S. Y. Savrasov, Phys. Rev. Lett. 81, 2570 (1998).

[10] For a review, see, e.g., S. Y. Savrasov, A. Toropova, M. I. Katsnelson, A. I. Lichtenstein, V. Antropov, G. Kotliar, Zeitsch. f. Krist. 220, 473 (2005).

[11] For a review, see, e.g., Strong Correlations in electronic structure calculations, ed. by V. I. Anisimov (Gordon and Breach Science Publishers, Amsterdam, 2000).

[12] For a review, see, e.g., F. Aryasetiawan, and O. Gunnarsson, Rep. Prog. Phys. 61, 237 (1998).

[13] I.V. Solovyev and K. Terakura, Phys. Rev. B 58, 15496 (1998).

[14] J. Hubbard, Proc. R. Soc. A 276, 238 (1963).

[15] F. C. Zhang and T. M. Rice, Phys. Rev. B 37, 3759 (1988).

[16] J. Hubbard, Phys. Rev. B 19, 2626 (1979), ibid. B 20 4584 (1979), ibid. B 235974 (1981).

[17] Y. Kakehashi, Phys. Rev. B 457196 (1992); B. L. Gyorffy, A. J. Pindor, J. Staunton, G. M. Stocks, H. Winter, J. Phys. F: Met. Phys. 15, 1337 (1985); ibid. 1387 (1985).

[18] For a recent review see G. Kotliar, S. Y. Savrasov, K. Haule, V. S. Oudovenko, O. Parcollet, and C. A. Marianetti, Rev. Mod. Phys. 78865 (2006).

[19] A. I. Lichtenstein and M. I. Katsnelson, and G. Kotliar, Phys. Rev. Lett. 87, 067205 (2001).

[20] K. Held, A. K. McMahan, and R. T. Scalettar, Phys. Rev. Lett. 87, 276404 (2001);

[21] S. Y. Savrasov, G. Kotliar and E. Abrahams, Nature (London) 410, 793 (2001). 
[22] V.I. Anisimov, A. I. Poteryaev, M. A. Korotin, A. O. Anokhin and G. Kotliar., J. Phys.: Condens. Matter 9, 7359 (1997); I. A. Nekrasov, K. Held, N. Blumer, A. I. Poteryaev, V. I. Anisimov, and D. Vollhardt, Eur. Phys. J. B 18, 55 (2000).

[23] M. I. Katsnelson and A.I. Lichtenstein, Phys. Rev. B 61, 8906 (2000);

[24] S.Y. Savrasov, K. Haule and G. Kotliar, Phys. Rev. Lett. 96, 036404 (2006).

[25] S.Y. Savrasov, Phys. Rev. B 54, 16470 (1996).

[26] A. Fujimori, F. Minami, and S. Sugano, Phys. Rev. B 29, 5225 (1984).

[27] J. van Elp, R. H. Potze, H. Eskes, R. Berger, and G.
Sawatzky, Phys. Rev. B 44, 1530 (1991).

[28] V.I. Anisimov, J. Zaanen and O.K. Andersen, Phys. Rev. B 44, 943 (1991).

[29] W.L. Roth, Phys. Rev. 110, 1333 (1958).

[30] D. Vaknin, E. Caignol, P. K. Davies, J. E. Fischer, D. C. Johnston, and D. P. Goshorn, Phys. Rev. B 39, 9122 (1989).

[31] K. Terakura, T. Oguchi, A. R. Williams, and Kübler, Phys. Rev. B 30, 4734, (1984).

[32] M.T. Hutchings and E.J. Samuelsen, Phys. Rev. B 6, 3447 (1972). 